\title{
Solvent Free Synthesis of Pyrimidine, Quinazolinone and Diazatricyclo Derivatives via Phase-Transfer Catalysis Method
}

\author{
AKBAR MOBINIKHALEDI ${ }^{1}$, NASER FOROUGHIFAR ${ }^{2}$, \\ TAHERE MOSLEH $^{1}$ and MAHSA JABBARPOUR ${ }^{1}$
}

${ }^{1}$ Department of Chemistry, Faculty of Science, Arak University, Arak 38156-8-8349, Iran

${ }^{2}$ Faculty of Chemistry, Tehran North Branch, Islamic Azad University, Tehran, Iran

akbar_mobini@yahoo.com

Received 30 July 2015 / Accepted 12 August 2015

\begin{abstract}
A simple and practical green chemistry procedure for the synthesis of some pyrimidine, quinazolinone and diazatricyclo derivatives is described. These compounds were synthesized in good to high yields by a Biginelli-like reaction of urea/thiourea, aldehyde and ketone under solvent free conditions in the presence of triethyl benzyl ammonium chloride (TEBAC) as a catalyst.
\end{abstract}

Keywords: Multicomponent, Biginelli, Solvent free, Catalyst, TEBAC

\section{Introduction}

Dihydropyrimidine-2-ones/thiones are an important class of heterocyclic compounds associated with a wide range of therapeutic and medicinal properties ${ }^{1,2}$ such as anti-microbial, antihypertensives, antiinflammatory ${ }^{3,4}$, antifungal ${ }^{5}$. They have been reported to show moderate calcium channel blocking ${ }^{6-8}$ and sodium channel blockade activities ${ }^{9}$. Also several marine alkaloids with interesting biological activities containing the dihydropyrimidine core have been known in the past few years ${ }^{10,11}$. Biginelli synthesized dihydropyrimidinones via a one-pot acid-catalyzed condensation reaction of $\beta$-ketoesters, aldehyds, and urea in refluxing ethanol ${ }^{12}$. However, this simple reaction is associated with low yield of products and prolongs reaction times. The importance of multicomponent reactions, MCRs, reactions and the interesting biological activities associated with dihydropyrimidinones led to the development of various multistep reactions that produce products in higher yields but lake of simplicity ${ }^{13-16}$.

Recently, a considerable attention has been focused on the synthesis of dihydropyrimidinones using more efficient methods ${ }^{17-24}$. In spite of their advantages, some of them need vigorous reaction conditions, expensive or unavailable catalyst, long reaction times and use of toxic solvents. Therefore, in order to overcome these limitations, the discovery and development of more practical and efficient protocols for the synthesis of 
Biginelli-like reactions is still of special interest for chemists. More recently, much attention has been directed toward the solvent-free reaction in order to develop classical methods and make them more clean and practical to carry out ${ }^{25,26}$.

In continuation of our work in synthesis of pyrimidines ${ }^{27-30}$ we wish to report the synthesis of pyrimidine, quinazolinone and diazatricyclo derivatives via a practical green chemistry procedure by using triethyl benzyl ammonium chloride (TEBAC) as a catalyst under solvent free conditions.

\section{Experimental}

All reagent and solvents used are commercially available. Melting points were measured with an Electrothermal apparatus. IR spectra were recorded with a Galaxy Series FT-IR 5000 spectrometer. The ${ }^{1} \mathrm{H}$ NMR and ${ }^{13} \mathrm{C}$ NMR spectra were recorded with a Bruker Avance $300 \mathrm{MHz}$ spectrometer with DMSO- $d_{6}$ as the solvent and TMS as the internal standard. The NMR spectra were identical with those of authentic materias ${ }^{30}$. Microanalyses were performed by the Elemental Analyzer (Elemental, Vario EL III) at the Arak University. Reactions were monitored by thin layer chromatography (TLC) using silica gel $\mathrm{F}_{254}$ aluminum sheets (Merck).

\section{General procedure for preparation of 4}

An equimolar amount of substituted aldehyde 1, urea or thiourea 2, ketone 3 and triethyl benzyl ammonium chloride (TEBAC, $5 \mathrm{~mol} \%$ ) was heated at $90{ }^{\circ} \mathrm{C}$ for an appropriate time. After completion of reaction, the reaction mixture was cooled to room temperature and ethanol was added $(10-15 \mathrm{~mL})$. The result solid was filtered, washed by hot water and ethanol and recrystallized from ethanol to give the pure product of 4a-n.

\section{Selected data}

\section{6-Methyl-4-phenyl-3,4-dihydropyrimidin-2(1H)-one (4a)}

Time: 130 min, Yield: 75\%, M.p 305-306 ${ }^{\circ} \mathrm{C}$. IR: $v 3303(\mathrm{NH}), 3223(\mathrm{NH}), 3068$ $\left(\mathrm{CH}_{\text {aromatic }}\right), 2972\left(\mathrm{CH}_{\text {aliphatic }}\right), 1656(\mathrm{C}=\mathrm{O}) .{ }^{1} \mathrm{H}$ NMR: $\delta 1.68\left(\mathrm{~s}, 3 \mathrm{H}, \mathrm{CH}_{3}\right), 4.92(\mathrm{~d}, J=9 \mathrm{~Hz}$, $\left.1 \mathrm{H}, \mathrm{H}_{\text {pyrimidine }}\right), 5.06(\mathrm{~d}, J=9 \mathrm{~Hz}, 1 \mathrm{H}, \mathrm{CH}), 6.61(\mathrm{~s}, 1 \mathrm{H}, \mathrm{NH}), 6.81(\mathrm{~s}, 1 \mathrm{H}, \mathrm{NH})$, the $\mathrm{NH}$ protons disappeared on $\mathrm{D}_{2} \mathrm{O}$ addition, , 7.04-7.45 (m, 5H, $\left.\mathrm{H}_{\text {aromatic }}\right) .{ }^{13} \mathrm{C}$ NMR (DMSO- $d_{6}, 75$ $\left.\mathrm{MHz}), \delta(\mathrm{ppm}) 43.8,49.9,126.7,127.7,128.0,128.8,142.0,4 \mathrm{H}, 2 \mathrm{CH}_{2}\right), 4.77(\mathrm{~s}, 1 \mathrm{H}$, $\left.\mathrm{H}_{\text {pyrimidine }}\right)$, 7.12-7.43 (m, 5H, $\left.\left.\mathrm{H}_{\text {aromatic }}\right), 8.76(\mathrm{~s}, 1 \mathrm{H}, \mathrm{NH}), 9.58(\mathrm{~s}, 1 \mathrm{H}, \mathrm{NH})\right)$, the $\mathrm{NH}$ protons disappeared on $\mathrm{D}_{2} \mathrm{O}$ addition. ${ }^{13} \mathrm{C}$ NMR: $\delta 23.8,26.8,54.2,58.2,125.3,127.4,128.3,128.7$, 129.0, 142.8, 143.0, 173.3. Anal cald for: $\mathrm{C}_{14} \mathrm{H}_{14} \mathrm{~N}_{2} \mathrm{OS}, \mathrm{C}, 65.09 ; \mathrm{H}, 5.46 ; \mathrm{N}, 10.84$; , $12.41 \%$. Found: C, 65.23; H, 5.48; N, 10.99; S, 12.30\%.

\section{5-Bromo-6-hydroxy-4,6-diphenyl-tetrahydropyrimidin-2(1H)-one (4e)}

Time: 100 min, Yield: 80\%, M.p 220-223 ${ }^{\circ} \mathrm{C}$. IR: v) 3217 (NH), 3190 (NH, OH), 3082 $\left(\mathrm{CH}_{\text {aromatic }}\right), 2980\left(\mathrm{CH}_{\text {aliphatic }}\right), 1682(\mathrm{C}=\mathrm{O}) .{ }^{1} \mathrm{H}$ NMR: $\delta 3.69\left(\mathrm{~d}, J=5.6 \mathrm{~Hz}, 1 \mathrm{H}, \mathrm{H}_{\text {pyrimidine }}\right), 5.04$ $(\mathrm{d}, J=5.6 \mathrm{~Hz}, 1 \mathrm{H}, \mathrm{CHBr}), 7.25-7.51\left(\mathrm{~m}, 11 \mathrm{H}, 10 \mathrm{H}_{\text {aromatic }}, 1 \mathrm{H}, \mathrm{OH}\right), 8.16(\mathrm{~s}, 1 \mathrm{H}, \mathrm{NH}), 8.82(\mathrm{~s}$, $1 \mathrm{H}, \mathrm{NH})$, the $\mathrm{NH}$ and $\mathrm{OH}$ protons disappeared on $\mathrm{D}_{2} \mathrm{O}$ addition. ${ }^{13} \mathrm{C}$ NMR: $\delta 20.5,55.5,66.4$, 123.9, 127.1, 128.0, 128.3, 128.9, 132.1, 132.8, 144.0, 153.7. Anal cald for: $\mathrm{C}_{16} \mathrm{H}_{15} \mathrm{BrN}_{2} \mathrm{O}_{2}, \mathrm{C}$, 55.35; H, 4.35; N, 8.07; Br, 23.01\%. Found: C, 55.45; H, 4.44; N, 8.15; Br, 23.21\%.

\section{6-Hydroxy-4,6-diphenyl-tetrahydropyrimidine-2(1H)-thione $(\mathbf{4 g})$}

Time: 90 min, Yield: 90\%, M.p 288-289 ${ }^{\circ} \mathrm{C}$. IR: $v 3387$ (NH), $3212(\mathrm{NH}, \mathrm{OH}), 3063\left(\mathrm{CH}_{\text {aromatic }}\right)$, $2947\left(\mathrm{CH}_{\text {aliphatic }}\right) .{ }^{1} \mathrm{H}$ NMR: $\delta 3.15\left(\mathrm{~d}, J=5.6 \mathrm{~Hz}, 2 \mathrm{H}, \mathrm{CH}_{2}\right), 4.15\left(\mathrm{t}, J=5.6 \mathrm{~Hz}, 1 \mathrm{H}, \mathrm{H}_{\text {pyrimidine }}\right)$, 6.97-6.99 (m, 5H, $\left.\mathrm{H}_{\text {aromatic }}\right), 7.13-7.15\left(\mathrm{~m}, 6 \mathrm{H}, 5 \mathrm{H}, \mathrm{H}_{\text {aromatic }}, 1 \mathrm{H}, \mathrm{OH}\right), 8.20(1 \mathrm{H}, \mathrm{NH}), 8.99(1 \mathrm{H}$, 
$\mathrm{NH}$ ), the $\mathrm{NH}$ and $\mathrm{OH}$ protons disappeared on $\mathrm{D}_{2} \mathrm{O}$ addition. ${ }^{13} \mathrm{C}$ NMR: $\delta 44.7,55.4,69.2,126.9$, $127.3,127.8,128.1,128.4,128.7,139.3,140.1,175.8$. Anal cald for: $\mathrm{C}_{16} \mathrm{H}_{16} \mathrm{~N}_{2} \mathrm{OS}, \mathrm{C}, 67.58 ; \mathrm{H}$, 5.67; N, 9.85; S, 11.28\%. Found: C, 67.78; H, 5.65; N, 9.71; S, 11.12\%.

\section{9-Methyl-8-oxa-10,12-diazatricyclo[7.3.1.0 $\left.0^{2,7}\right]$ trideca-2,4,6-triene-11-one (4h)}

Time: 80 min, Yield: 90\%, M.p 291-293 ${ }^{\circ} \mathrm{C}$. IR: v $3311(\mathrm{NH}), 3240(\mathrm{NH}), 3120\left(\mathrm{CH}_{\text {aromatic }}\right)$, $2891\left(\mathrm{CH}_{\text {aliphatic }}\right), 1687(\mathrm{C}=\mathrm{O}) .{ }^{1} \mathrm{H}$ NMR: $\delta 1.58\left(\mathrm{~s}, 3 \mathrm{H}, \mathrm{CH}_{3}\right), 2.09\left(\mathrm{~d}, J=5.6 \mathrm{~Hz}, 2 \mathrm{H}, \mathrm{CH}_{2}\right)$, $4.22\left(\mathrm{t}, J=5.6 \mathrm{~Hz}, 1 \mathrm{H}, \mathrm{H}_{\text {pyrimidine }}\right), 6.72-7.13\left(\mathrm{~m}, 4 \mathrm{H}, \mathrm{H}_{\text {aromatic }}\right), 7.28(\mathrm{~s}, 1 \mathrm{H}, \mathrm{NH}), 7.58(\mathrm{~s}, 1 \mathrm{H}$, $\mathrm{NH}$ ), the $\mathrm{NH}$ protons disappeared on $\mathrm{D}_{2} \mathrm{O}$ addition. ${ }^{13} \mathrm{C}$ NMR: $\delta 26.7,32.6,44.9,82.6$, $116.9,120.5,126.0,129.2,129.5,151.7,155.7$. Anal cald for: $\mathrm{C}_{11} \mathrm{H}_{12} \mathrm{~N}_{2} \mathrm{O}_{2}, \mathrm{C}, 64.69 ; \mathrm{H}$, $5.92 ; \mathrm{N}, 13.72 \%$. Found: C, 64.48; H, 6.04; N, 13.51\%.

\section{9-Phenyl-8-oxa-10,12-diazatricyclo [7.3.1.0 $\left.0^{2,7}\right]$ trideca-2,4,6-triene-11-one (4I)}

Time: 105 min, Yield: 87\%, M.p 272-273 ${ }^{\circ} \mathrm{C}$. IR: $v 3321(\mathrm{NH}), 3219(\mathrm{NH}), 3072$ $\left(\mathrm{CH}_{\text {aromatic }}\right), 2982\left(\mathrm{CH}_{\text {aliphatic }}\right), 1687(\mathrm{C}=\mathrm{O}) .{ }^{1} \mathrm{H}$ NMR: $\delta 2.20\left(\mathrm{~d}, J=5.6 \mathrm{~Hz}, 2 \mathrm{H}, \mathrm{CH}_{2}\right), 4.32$ (t, $\left.J=5.6 \mathrm{~Hz}, 1 \mathrm{H}, \mathrm{H}_{\text {pyrimidine }}\right), 6.80-7.88\left(\mathrm{~m}, 9 \mathrm{H}, \mathrm{H}_{\text {aromatic }}\right), 8.64(\mathrm{~s}, 1 \mathrm{H}, \mathrm{NH}), 9.71(\mathrm{~s}, 1 \mathrm{H}, \mathrm{NH})$, the $\mathrm{NH}$ protons disappeared on $\mathrm{D}_{2} \mathrm{O}$ addition. ${ }^{13} \mathrm{C}$ NMR: $\delta 35.3,49.9,85.2,121.0,125.7,126.2$, $126.8,128.3,128.7,129.5,131.2,134.6,151.8,156.3$. Anal cald for: $\mathrm{C}_{16} \mathrm{H}_{14} \mathrm{~N}_{2} \mathrm{O}_{2}, \mathrm{C}, 72.16$; H, 5.30; N, 10.52\%. Found: C, 72.37; H, 5.25; N, 10.68\%.

\section{9-(4-Bromophenyl)-8-oxa-10,12-diazatricyclo[7.3.1.0 $0^{2,7}$ trideca-2,4,6-triene-11-one (4n)}

Time: 115 min, Yield: 86\%, M.p 285-287 ${ }^{\circ} \mathrm{C}$. IR: $v 3317$ (NH), $3220(\mathrm{NH}), 3074$ $\left(\mathrm{CH}_{\text {aromatic }}\right), 2914\left(\mathrm{CH}_{\text {aliphatic }}\right), 1682(\mathrm{C}=\mathrm{O}) .{ }^{1} \mathrm{H}$ NMR: $\delta \delta(\mathrm{ppm}) 2.17(\mathrm{~d}, J=5.6 \mathrm{~Hz}, 2 \mathrm{H}$, $\left.\mathrm{CH}_{2}\right), 4.31\left(\mathrm{t}, J=5.6 \mathrm{~Hz}, 1 \mathrm{H}_{\text {pyrimidine }}\right), 6.92-7.26\left(\mathrm{~m}, 4 \mathrm{H}, \mathrm{H}_{\text {aromatic }}\right), 7.40-7.62(\mathrm{~m}, 4 \mathrm{H}$, $\left.\mathrm{H}_{\text {aromatic }}\right), 7.64(\mathrm{~s}, 1 \mathrm{H}, \mathrm{NH}), 7.90(\mathrm{~s}, 1 \mathrm{H}, \mathrm{NH})$, the $\mathrm{NH}$ protons disappeared on $\mathrm{D}_{2} \mathrm{O}$ addition. ${ }^{13}$ C NMR: $\delta$ 35.2, 45.1, 85.0, 121.1, 122.2, 126.0, 128.7, 129.4, 129.7, 131.5, 131.7, 141.3, 151.4, 156.1. Anal cald for: $\mathrm{C}_{16} \mathrm{H}_{13} \mathrm{BrN}_{2} \mathrm{O}_{2}, \mathrm{C}, 55.67 ; \mathrm{H}, 3.80 ; \mathrm{N}, 8.12 ; \mathrm{Br}, 23.15 \%$. Found: C, 55.89; H, 3.85; N, 7.98; Br, 23.32\%.

\section{Results and Discussion}

The synthetic pathway for the synthesis of desired compounds 4a-n is presented in Scheme 1.

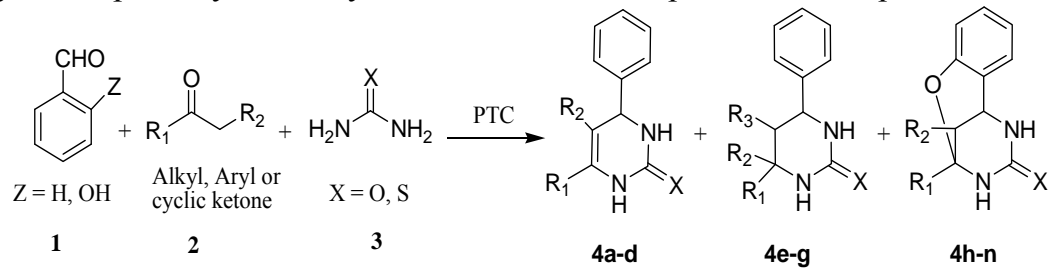

$$
\begin{aligned}
& \text { 4a: } X=\mathrm{O}, \mathrm{R}_{1}=\mathrm{CH}_{3}, \mathrm{R}_{2}=\mathrm{H} \\
& \text { 4b: } X=\mathrm{S}, \mathrm{R}_{1}=\mathrm{CH}_{2} \mathrm{CH}_{2}, \mathrm{R}_{2}=\mathrm{CH}_{2} \mathrm{CH}_{2} \\
& \text { 4c: } X=\mathrm{O}, \mathrm{R}_{1}=\mathrm{CH}_{2} \mathrm{CH}_{2}, \mathrm{R}_{2}=\mathrm{CH}_{2} \mathrm{CO} \\
& \text { 4d: } X=\mathrm{S}, \mathrm{R}_{1}=\mathrm{CH}_{2} \mathrm{CH}_{2}, \mathrm{R}_{2}=\mathrm{CH}_{2} \mathrm{CO} \\
& \text { 4e: } X=\mathrm{O}, \mathrm{R}_{1}=\mathrm{C}_{6} \mathrm{H}_{5}, \mathrm{R}_{2}=\mathrm{OH}, \mathrm{R}_{3}=\mathrm{Br} \\
& \text { 4f: } X=\mathrm{S}, \mathrm{R}_{1}=\mathrm{CH}_{3}, \mathrm{R}_{2}=\mathrm{OH}, \mathrm{R}_{3}=\mathrm{H} \\
& \text { 4g: } X=\mathrm{S}, \mathrm{R}_{1}=\mathrm{C}_{6} \mathrm{H}_{5}, \mathrm{R}_{2}=\mathrm{OH}, \mathrm{R}_{3}=\mathrm{H}
\end{aligned}
$$

$$
\begin{aligned}
& \text { 4h: } X=O, R_{1}=C_{3}, R_{2}=H \\
& \text { 4i: } X=S, R_{1}=C_{3}, R_{2}=H \\
& \text { 4j: } X=O, R_{1}=C_{2} C_{2}, R_{2}=C_{2} C_{2} H_{2} \\
& \text { 4k: } X=S, R_{1}=C_{2} C_{2}, R_{2}=C_{2} C_{2} \\
& \text { 4l: } X=O, R_{1}=C_{6} H_{5}, R_{2}=H \\
& \text { 4m: } X=S, R_{1}=C_{6} H_{5}, R_{2}=H \\
& \text { 4n: } X=O, R_{1}=4 B r-C_{6} H_{4}, R_{2}=H
\end{aligned}
$$

Scheme 1. Synthetic pathway of pyrimidine, quinazolinone and diazatricyclo derivatives 4a-n

Reaction of substituted aldehyde 1 with urea or thiourea and corresponding ketone in the presence triethyl benzyl ammonium chloride (TEBAC) as a catalyst gave compounds 4a-n. This Biginelli-like reaction was carried out with different ketones. In the case of salicylaldehyde as a 
starting material, the Biginelli-like reaction is followed by a Michael addition and formation of an oxygen bridge with C-6 of the pyrimidine ring to produce diazatricyclo derivatives $4 \mathbf{h}-\mathbf{n}$. The formation of this oxygen bridge may be due to the effective distance between the orthohydroxyl group of phenol group and the C-6 atom of pyrimidine ring ${ }^{31}$.

The good to high yields of reactions, use of safe and available catalyst and green reaction conditions are some advantages of this method for the synthesis of desired compounds. All synthesized compounds were identified using IR, NMR spectroscopy as well as elemental analysis and also by comparison with their authentic samples ${ }^{31}$. In the ${ }^{1} \mathrm{H}$ NMR spectrum of 4a-n, the appearance of two broad singlets at $\sim 6.60-9.70 \mathrm{ppm}$ is related to the resonance of two $\mathrm{NH}$ protons (disappeared on $\mathrm{D}_{2} \mathrm{O}$ addition). Also, in the IR spectra of these compounds the appearance of the absorption bond at $\sim 3184-3421 \mathrm{~cm}^{-1}$, the characteristic of the NH group, are good evidences in support of expected reactions

\section{Acknowledgement}

We gratefully acknowledge the Chemistry Department of Arak University for providing of financial supports.

\section{References}

1. Kappe C O, Eur J Med Chem., 2000, 35(12), 1043-1052; DOI:10.1016/S02235234(00)01189-2

2. Atwal K S, Rovnyak G. C, Kimball S D, Floyd D M, Moreland S, Swanson B N, Gougoutas J Z, Schwart J, Smillie K M and Malley M F, J Med Chem., 1990, 33(9), 2629-2635; DOI:10.1021/jm00171a044

3. Grover G. J, Dzwonczyk S, McMullen D M, Normandin D E, Parham C S, Sleph P G and Moreland S, J Cardiovasc Pharmacol., 1995, 26(2), 289-294.

4. Kappe C O, Tetrahedron, 1993, 49(32), 6937-6963; DOI:10.1016/S00404020(01)87971-0

5. Singh O M, Singh S J, Devi M B, Devi L N, Singh N I and Lee S G, Bioorg Med Chem Lett., 2008, 18(24), 6462-6467; DOI:10.1016/j.bmcl.2008.10.063

6. Singh K, Arora D, Poremsky E, Lowery J and Moreland R S, Eur J Med Chem., 2009, 44(5), 1997-2001; DOI:10.1016/j.ejmech.2008.10.002

7. Kshirsagar S S and Shanmugasundaram P, Chem Tech., 2013, 5, 2899-2912

8. Rovnyak G C, Kimball S D, Beyer B, Cucinotta G, DiMarco J D, Gougoutas J, Hedberg A, Malley M, McCarthy J P, Zhang R and Moreland S, J Med Chem., 1995, 38(1), 119-129; DOI:10.1021/jm00001a017

9. Snider B B and Shi Z, J Org Chem., 1993, 58(15), 3828-3839; DOI:10.1021/jo00067a014

10. Zhen Y S, Qi C Q, Hu J L, Xue Y C and Chen W J, Adv Exp Med Biol., 1998, 431, 575-579.

11. Biginelli P, Gazz Chim Ital., 1893, 23, 360-413.

12. Barluenga J, Tomas M, Ballesteros A and Lopez L A, Tetrahedron Lett., 1989, 30(34), 4573-4576; DOI:10.1016/S0040-4039(01)80748-6

13. Wipf $\mathrm{P}$ and Cunningham A, Tetrahedron Lett., 1995, 36(43), 7819-7822; DOI:10.1016/0040-4039(95)01660-A

14. O'Reilly B C and Atwal K S, Heterocycles, 1987, 26, 1185-1188.

15. Shaabani A, Sarvary A, Rahmati A and Rezayan A H, Lett Org Chem., 2007, 4(1), 68-71; DOI:10.2174/157017807780037531 
16. Dwivedi N, Mishra R C and Tripathi R P, Lett Org Chem., 2005, 2(5), 450-457; DOI:10.2174/1570178054405986

17. Mobinikhaledi A, Foroughifar N, Karimi G and Foroughifar N, Synth React Inorg Met Org Nano Metal Chem., 2007, 37(4), 279-282;

DOI:10.1080/15533170701316809

18. Pansuriya A M, Savant M M, Bhuva C V, Singh J and Naliapara Y T, ARKIVOC. 2009, 7, 79-85.

19. Panchasara D and Pande S, J Chem., 2009, 6(S1), S91-S96;

DOI: $10.1155 / 2009 / 457168$

20. Pourjavadi A, Salimi H, Barzegar S and Eftekhari-Sis B, Acta Chim Slov., 2007, 54, 140-143.

21. Lin H, Ding J, Chen X and Zhang Z, Molecules, 2000, 5(12), 1240-1243; DOI: $10.3390 / 51201240$

22. Kappe C O and Roschger P, J Heterocyclic Chem., 1989, 26(1), 55-64; DOI:10.1002/jhet.5570260112

23. Rodríguez-Domínguez J C, Bernardi D and Kirsch G, Tetrahedron Lett., 2007, 48(33), 5777-5780; DOI:10.1016/j.tetlet.2007.06.104

24. Oskooie H A, Heravi M M, Karimi N and Monjezy M H, Synth Commun., 2011, 41, 826-831.

25. Mobinnikhaledi A, Foroughifar N, Alipour Safari J and Amini E, $J$ Heterocyclic Chem., 2007, 44(3), 697-699; DOI:10.1002/jhet.5570440329

26. Foroughifar N, Mobinikhaledi A and Fathinejad Jirandehi H, Phosphorus Sulfur Silicon Relat Elem., 2003, 178, 495-500.

27. Foroughifar N, Mobinikhaledi A and Fathinejad Jirandehi H, Phosphorus, Sulfur Silicon Relat Elem., 2003, 178, 1241-1246.

28. Mobinikhaledi A, Foroughifar N and Fathinejad Jirandehi H, Phosphorus, Sulfur Silicon Relat Elem.. 2004, 179(11), 2259-2263.

29. Zendehdel M, Mobinikhaledi A and Asgari A, J Incl Phenom Macro., 2008, 60(3-4), 353-357; DOI:10.1007/s10847-007-9384-2

30. Akbar Mobinikhaledi, Naser Foroughifar, Tahere Mosleh and Ahmad Hamta, Phosphorus, Sulfur Silicon, 2012, 187(6), 728-734.

31. Abbas E M H, Abdallah S M, Abdoh M H, Tawfik H A and El-Hamouly W S, Turk $J$ Chem., 2008, 32, 297-304. 